\title{
Long-term survival and prognostic implications of Chinese type 2 diabetic patients with coronary artery disease after coronary artery bypass grafting
}

\author{
Ming C Hsiung ${ }^{1}$, Tao-Hsin Tung ${ }^{2}$, Chung-Yi Chang ${ }^{3}$, Yi-Cheng Chuang ${ }^{3}$, Kuo-Chen Lee ${ }^{3}$, \\ Sung-How Sue ${ }^{3}$, Yi-Pen Chou ${ }^{4}$, Richard Hsiung ${ }^{1}$, Chien-Ming Huang ${ }^{1}$, Chang-Chyi Lin ${ }^{1}$, \\ Wei-Hsian Yin ${ }^{1}$, Mason S Young ${ }^{1}$, Jeng $\mathrm{Wei}^{3^{*}}$ \\ ${ }^{1}$ Division of Cardiology, Department of Medicine, Cheng-Hsin General Hospital, Taipei, Taiwan \\ ${ }^{2}$ Department of Medicine Research and Education, Cheng-Hsin General Hospital, Taipei, Taiwan \\ ${ }^{3}$ Division of Cardiovascular Surgery, Cheng-Hsin General Hospital, Taipei, Taiwan; ch2876@echgh.org.tw \\ ${ }^{4}$ Department of Anesthesiology, Cheng-Hsin General Hospital, Taipei, Taiwan \\ *Corresponding author
}

Received 13 August 2009; revised 11 September 2009; accepted 14 September 2009.

\begin{abstract}
This study was conducted to determine the survival rates of type 2 diabetic patients after coronary artery bypass grafting (CABG) surgery, and the prognostic factors related to all-cause mortality during a 7-year follow-up. The medical records of 816 type 2 diabetic patients who underwent primary, isolated CABG surgery during 1997-2003 were studied. The Kaplan-Meier method and Cox regression were used to estimate survival and the independence of prognostic factors associated with all-cause mortality. One hundred and five out of 816 patients expired during the 7-year period. The overall survival rate was $83.5 \%(95 \% \mathrm{Cl}: 78.7-88.3 \%)$. Associated prog- nostic factors varied by gender. For males, but not for females, smoking $(R R=3.44,95 \% \mathrm{Cl}$ : 1.01- 11.70), myocardial infarction ( $R R=4.77$, $95 \% \mathrm{Cl}: 1.23-18.86)$, respiratory failure $(R R=4.21$, 95\% Cl: 1.48-7.99), cardiogenic shock ( $R R=12.58$, 95\% Cl: 3.25-20.77), and congestive heart failure $(R R=3.77,95 \% \mathrm{Cl}: 1.10-13.45)$ were significantly related to all-cause mortality. Older age at surgery $(R R=1.15,95 \% \mathrm{Cl}: 1.04-1.27)$, emergent operation ( $R R=21.29,95 \% \mathrm{Cl}$ : $10.03-39.43$ ), pulmonary ede-ma (RR=7.72, $95 \% \mathrm{Cl}$ : $1.89-15.59$ ), and days of ICU stay (RR=1.05, 95\% CI: 1.02-1.09) were significant- ly related to all-cause mortality in females only. In conclusion, several gender-related differences were noted pertaining to all-cause mortality and its relationship to smoking, myocardial infarction, respiratory failure, cardiogenic shock, congestive heart failure, older age at surgery, emergent operation, pulmonary edema, and days of ICU stay.
\end{abstract}

Keywords: Coronary Artery Bypass Grafting; Gender Difference; Prognostic Factors; Survival; Type 2 Diabetes

\section{INTRODUCTION}

Diabetes mellitus is viewed as a major risk factor for cardiovascular disease, and coronary heart disease is the leading cause of death in adult diabetics [1]. In general, around $20 \%$ of patients undergoing coronary artery bypass surgery (CABG) suffer from diabetes mellitus [1]. In addition, diabetics have a higher mortality than patients without diabetes after CABG surgery [2,3]. Choosing this type of surgical therapy for patients depends on the estimate of the risk of the procedure and the possible benefits compared with alternative forms of therapy. Long-term outcome among diabetics with coronary artery disease (CAD) after CABG is associated with severity of diabetes at the time of surgery [4]. This observation suggests that early detection of this disorder followed by early treatment could improve both survival and quality of life.

In Taiwan, the remarkable economic achievements of the last 40 years have resulted in a great improvement in living standards and in an increase in life expectancy. Type 2 diabetes in adults is one of the global public health problems in the Taiwanese population. Screening for type 2 diabetes by the natural history of diabetes, fasting plasma glucose (FPG), and oral glucose tolerance test (OGTT) showed the overall prevalence of type 2 diabetes in Taiwan to be $5-9 \%$ and $11-13 \%$ for residents above 20 or 40 years of age, respectively [5-11]. From the viewpoint of evidence-based medicine, it is not only important to be cognizant of the long-term survival of diabetic patients after $\mathrm{CABG}$, but also to explore the complete spectrum of demographic and biological markers that 
may be related to survival rate. To the best of our knowledge, the long-term survival of Chinese type 2 diabetic patients with $\mathrm{CAD}$ undergoing $\mathrm{CABG}$ surgery has not been carefully examined. Some uncertainty still exists regarding the influence of gender on long-term survival and on associated risk factors for long-term survival for these patients after CABG surgery. Thus, in order to determine the long-term survival of and associated risk factors for diabetic patients after CABG surgery, a longterm survival study was performed.

Because long-term prognosis after CABG surgery in relation to gender has not been closely evaluated among type 2 diabetic patients in Taiwan, the present study was designed to explore the possibility of gender-related differences in risk factors, because such differences might have important implications for understanding the value of individual prognostic factors for estimation of longterm survival of diabetic patients after surgery. The purpose of this study was to determine the long-term (7-year follow-up) survival rate of each gender after primary, isolated CABG surgery, and to identify the associated prognostic factors related to all-cause mortality among Chinese type 2 diabetic patients.

\section{MATERIALS AND METHODS}

\subsection{Patient Population}

Between January 1997 and December 2003, a total of 898 type 2 diabetic patients with CAD underwent CABG surgery at the Division of Cardiovascular Surgery and Heart Center, Cheng-Hsin General Hospital, a fully certified regional and teaching hospital with 757 beds in Taipei, Taiwan. Of the 898 type 2 diabetic patients, 66 $(7.3 \%)$ were excluded due to simultaneous valve surgery and another $16(1.8 \%)$ patients were excluded due to repeated $\mathrm{CABG}$ surgery. The remaining 816 patients who underwent primary, isolated CABG surgery because of $\mathrm{CAD}$ without concomitant valve procedures and without previous history of CABG participated in the evaluation. Seventy-one per cent $(581 / 816)$ of these participants were males.

\subsection{Data Collection}

Patients' demographic characteristics and surgical variables were retrieved from medical records and computer files. The hospital records of each diabetic patient were reviewed by well trained, senior chart reviewers using a standard data collection form. Demographic information, presence of pre-existing co-morbidities and associated medications at admission and discharge were obtained from the nursing and medical histories. Indicators of postoperative recovery and complications were identified from the details of patient's progress notes. Subjects were divided into three categories according to smoking or alcohol drinking: current users, ex-users, and non users. We combined current users and ex-users as the user group. The identification of type 2 diabetes was based on the WHO definition in 1999 [12]: subjects with $F P G \geq 126$ $\mathrm{mg} / \mathrm{dl}$ or $2 \mathrm{~h}$ post-load $\geq 200 \mathrm{mg} / \mathrm{dl}$ were defined as individuals with type 2 diabetes. Hypertension was defined as systolic blood pressure $\geq 140 \mathrm{mmHg}$ or diastolic blood pressure $\geq 90 \mathrm{mmHg}$ [13]. Hyperlipidaemia was defined as one or more of the following: total cholesterol $\geq 200$ $\mathrm{mg} / \mathrm{dl}$; triglyceride $\geq 200 \mathrm{mg} / \mathrm{dl} ; \mathrm{HDL}<35 \mathrm{mg} / \mathrm{dl}$ [14]. Subjects featuring a personal disease history of type 2 diabetes, hypertension, or hyperlipidaemia and who had received medication to treat such conditions were viewed as "known cases" of such disease. In addition, for diabetic patients after CABG who died in the hospital, case records such as autopsy findings were used for information about the causes of death. For those patients who died outside the hospital, information including autopsy findings, scheduled investigations, and death certificates were collected. In addition, access to hospital records was approved by the hospital human subjects review board at Cheng-Hsin General Hospital.

\subsection{Operative Technique}

The operative procedures for CABG surgery have been described in previous studies [15-17]. The CABG operations were performed by means of a median sternotomy with the aid of extracorporeal cardiopulmonary bypass hemodilution (hematocrit, $25 \%$ to $30 \%$ ) and moderate hypothermia $\left(25\right.$ to $28^{\circ} \mathrm{C}$ ). Cardiopulmonary bypass used an ascending aortic cannula followed by a two-stage venous cannula. Myocardial preservation was achieved with a hypercalcemic, hypothermic crystalloid solution and external cooling with ice slush or a cold saline solution. Crystalloid cardioplegia was infused through the aortic root at least every 20 minutes and was infused into each completed vein graft in addition to the aortic root infusion. Blood cardioplegia was usually used for patients with left ventricular hypertrophy, cardiogenic shock, or acutely occluded coronary arteries. Venting was performed primarily via the aorta, and if necessary, via the pulmonary artery. The myocardial temperature was monitored and kept below $15^{\circ} \mathrm{C}$. After completion of all distal anastomoses, the patient was warmed systemically to $37^{\circ} \mathrm{C}$, while warm cardioplegic solution followed by warm blood was given by the antegrade method.

\subsection{Statistical Analysis}

Statistical analysis was performed using SAS software. Prognostic predictors and all-cause mortality were determined by univariate and multivariate techniques. In the univariate analysis, chi-square testing or two-sample 
Table 1. Comparison of baseline characteristics among type 2 diabetic patients with CAD after CABG surgery $(n=816)$.

\begin{tabular}{|c|c|c|c|c|c|c|c|c|}
\hline & & \multicolumn{2}{|c|}{$\begin{array}{c}\text { Male } \\
(\mathrm{n}=581)\end{array}$} & \multicolumn{2}{|c|}{$\begin{array}{l}\text { Female } \\
(\mathrm{n}=235)\end{array}$} & \multicolumn{2}{|c|}{$\begin{array}{c}\text { Total } \\
(\mathrm{n}=816)\end{array}$} & \multirow{2}{*}{$\begin{array}{c}\mathrm{p} \text {-value for } \mathrm{t} \text {-test or } \\
\chi^{2} \text {-test }\end{array}$} \\
\hline & & $\mathrm{n}$ & $(\%)$ & $\mathrm{n}$ & $(\%)$ & $\mathrm{n}$ & $(\%)$ & \\
\hline \multicolumn{9}{|l|}{ Categorical variables } \\
\hline Known case of diabetes & 385 & & $(66.3)$ & 165 & $(70.2)$ & 550 & $(67.4)$ & 0.28 \\
\hline Emergent operation & 2 & & $(0.3)$ & 3 & $(1.3)$ & 5 & $(0.6)$ & 0.12 \\
\hline Smoking & 160 & & $(27.5)$ & 27 & $(11.5)$ & 187 & $(22.9)$ & $<0.0001$ \\
\hline Alcohol drinking & 82 & & $(14.1)$ & 16 & $(6.8)$ & 98 & $(12.0)$ & 0.005 \\
\hline MI (Inferior or Posterior) & 227 & & $(39.1)$ & 77 & $(32.7)$ & 304 & $(37.3)$ & 0.09 \\
\hline Hypertension & 417 & & $(71.8)$ & 185 & $(78.7)$ & 602 & $(73.8)$ & 0.04 \\
\hline Hyperlipidemia & 227 & & $(39.1)$ & 90 & $(38.3)$ & 317 & (38.9) & 0.84 \\
\hline COPD & 36 & & $(6.2)$ & 14 & $(6.0)$ & 50 & $(6.1)$ & 0.90 \\
\hline PAOD & 24 & & $(4.1)$ & 16 & $(6.8)$ & 40 & $(4.9)$ & 0.11 \\
\hline Pulmonary edema & 53 & & $(9.1)$ & 32 & $(13.6)$ & 85 & $(10.4)$ & 0.06 \\
\hline Old CVA & 74 & & $(12.7)$ & 32 & $(13.6)$ & 106 & $(13.0)$ & 0.73 \\
\hline Renal failure & 82 & & $(14.1)$ & 49 & $(20.9)$ & 131 & $(16.1)$ & 0.02 \\
\hline Sepsis & 8 & & $(1.4)$ & 6 & $(2.6)$ & 14 & $(1.7)$ & 0.24 \\
\hline UGI bleeding & 9 & & (1.6) & 4 & $(1.7)$ & 13 & (1.6) & 0.87 \\
\hline Respiratory failure & 26 & & $(4.5)$ & 10 & $(4.3)$ & 36 & $(4.5)$ & 0.93 \\
\hline Cardiogenic shock & 39 & & $(6.7)$ & 11 & $(4.7)$ & 50 & $(6.1)$ & 0.30 \\
\hline Unstable angina & 194 & & $(33.4)$ & 92 & $(39.1)$ & 286 & $(35.0)$ & 0.08 \\
\hline Pre PTCA & 102 & & $(17.6)$ & 31 & $(13.2)$ & 133 & $(16.3)$ & 0.16 \\
\hline $\mathrm{CHF}$ & 62 & & $(10.7)$ & 25 & $(10.6)$ & 87 & $(10.7)$ & 0.94 \\
\hline Type of bypass (arterial/venous) & $464 / 117$ & & $\begin{array}{l}(79.9 \\
/ 20.1)\end{array}$ & $162 / 73$ & $\begin{array}{l}(68.9 \\
/ 31.1)\end{array}$ & $626 / 190$ & $\begin{array}{l}(76.7 \\
/ 23.3)\end{array}$ & 0.001 \\
\hline \multicolumn{9}{|l|}{ Continuous variables } \\
\hline Duration of diabetes & \multicolumn{3}{|c|}{$12.64 \pm 4.85$} & \multicolumn{2}{|c|}{$14.78 \pm 5.32$} & \multicolumn{2}{|c|}{$13.57 \pm 4.97$} & 0.01 \\
\hline Operation age (yrs) & \multicolumn{3}{|c|}{$64.02 \pm 10.05$} & \multicolumn{2}{|c|}{$66.50 \pm 8.18$} & \multicolumn{2}{|c|}{$64.74 \pm 9.61$} & 0.0003 \\
\hline FPG (mg/dl) & \multicolumn{3}{|c|}{$169.1 \pm 107.6$} & \multicolumn{2}{|c|}{$201.2 \pm 106.6$} & \multicolumn{2}{|c|}{$178.4 \pm 98.2$} & 0.002 \\
\hline HbA1c (\%) & \multicolumn{3}{|c|}{$8.2 \pm 2.0$} & \multicolumn{2}{|c|}{$8.4 \pm 1.9$} & \multicolumn{2}{|c|}{$8.2 \pm 2.0$} & 0.54 \\
\hline $\mathrm{SBP}(\mathrm{mmHg})$ & \multicolumn{3}{|c|}{$135.0 \pm 20.7$} & \multicolumn{2}{|c|}{$141.1 \pm 22.2$} & 136 & 21.4 & 0.02 \\
\hline $\mathrm{DBP}(\mathrm{mmHg})$ & & 76.7 & 11.9 & & 3.8 & & 12.6 & 0.11 \\
\hline BMI $\left(\mathrm{Kg} / \mathrm{m}^{2}\right)$ & & 26.7 & 17.8 & & & & 15.0 & 0.15 \\
\hline Cholesterol (mg/dl) & & 189.3 & 44.3 & 204 & 31.8 & 193 & 50.0 & 0.002 \\
\hline Triglyceride (mg/dl) & & 190.2 & 147.0 & 20 & 60.4 & 194 & 142.8 & 0.32 \\
\hline BUN (mg/dl) & & 23.4 & 17.6 & & 5.0 & & 16.8 & 0.56 \\
\hline Creatinine (mg/dl) & & 1.70 & 1.92 & & .68 & & 0.85 & 0.63 \\
\hline Uric acid (mg/dl) & & 7.5 & & & & & & 0.91 \\
\hline AST (U/L) & & 50.0 & 57.0 & & 1.5 & & 64.8 & 0.37 \\
\hline $\operatorname{ALT}(\mathrm{U} / \mathrm{L})$ & & $40.6 \pm$ & 07.0 & & 5.5 & & 91.6 & 0.19 \\
\hline $\mathrm{HDL}(\mathrm{mg} / \mathrm{dl})$ & & 38.5 & 11.7 & & 7.5 & & 36.7 & 0.09 \\
\hline LDL (mg/dl) & & 125.4 & 38.0 & 131 & 48.1 & 127 & 41.0 & 0.18 \\
\hline $\operatorname{RBC}\left(10^{3} / \mathrm{ul}\right)$ & & 4.3 & & & & & & 0.28 \\
\hline WBC $\left(10^{3} / \mathrm{ul}\right)$ & & 9.4 & & & 5.4 & & 40.6 & 0.10 \\
\hline Hs-CRP(mg/dl) & & 0.189 & 0.177 & 0.17 & .164 & 0.18 & 0.152 & 0.51 \\
\hline Ischaemic time (min) & & 61.64 & 41.01 & 65.1 & 8.65 & 62.6 & 40.35 & 0.26 \\
\hline Cardiopulmonary bypass time (min) & & 104.99 & 68.65 & 111. & 67.68 & 106. & 688.40 & 0.19 \\
\hline CAD number & & 2.83 & 0.44 & & .54 & & 0.47 & 0.02 \\
\hline CABG number & & 3.13 & 0.97 & & .02 & & 0.99 & 0.12 \\
\hline Pre-operation LVEF (\%) & & 50.93 & 19.68 & 54. & 9.91 & 51.8 & 19.78 & 0.03 \\
\hline Post-operation LVEF (\%) & & 55.25 & 17.24 & 57.7 & 8.58 & 56.0 & 17.68 & 0.17 \\
\hline ICU stay (days) & & 6.44 & 7.17 & 8.6 & 3.88 & & 9.64 & 0.02 \\
\hline
\end{tabular}

CAD: coronary artery disease, CABG: coronary artery bypass grafting, MI: myocardial infarction, COPD: chronic obstructive pulmonary disease, PAOD: peripheral arterial occlusive disease, CVA: cerebrovascular accident, PTCA: percutaneous transluminal coronary angioplasty, CHF: congestive heart failure. 
Table 2. Multivariate analysis using Cox regression model of risk factors associated with the all-cause mortality that all univariatesignificant factors were included among type 2 diabetic patients with CAD after CABG surgery.

\begin{tabular}{|c|c|c|c|c|c|c|}
\hline \multirow[b]{3}{*}{ Variable } & \multicolumn{6}{|c|}{ All causes of death (yes vs no) } \\
\hline & \multicolumn{2}{|c|}{ Male } & \multicolumn{2}{|c|}{ Female } & \multicolumn{2}{|c|}{ Total } \\
\hline & RR & $(95 \% \mathrm{CI})$ & $\mathrm{RR}$ & $(95 \% \mathrm{CI})$ & $\mathrm{RR}$ & $(95 \% \mathrm{CI})$ \\
\hline Gender (male vs female) & ----- & ---- & ----- & ---- & 0.26 & $(0.13-0.54)$ \\
\hline Operation age (yrs) & 1.03 & $(0.97-1.10)$ & 1.15 & $(1.04-1.27)$ & 1.05 & $(1.00-1.10)$ \\
\hline Emergent operation (yes vs no) & 10.56 & $(0.67-20.06)$ & 21.29 & $(10.03-39.43)$ & 12.45 & $(0.21-22.77)$ \\
\hline Smoking (yes vs no) & 3.44 & $(1.01-11.70)$ & 2.56 & $(0.35-18.48)$ & 2.03 & $(0.82-5.00)$ \\
\hline Myocardial infraction (yes vs no) & 4.77 & $(1.23-18.86)$ & 1.46 & $(0.13-2.83)$ & 3.31 & $(1.27-6.80)$ \\
\hline Pulmonary edema (yes vs no) & 1.55 & $(0.53-2.39)$ & 6.56 & $(1.22-15.21)$ & 2.00 & $(0.33-3.51)$ \\
\hline Sepsis (yes vs no) & 6.50 & $(0.61-12.41)$ & 4.97 & $(0.19-19.81)$ & 5.67 & $(0.42-13.27)$ \\
\hline Respiratory failure (yes vs no) & 4.21 & $(1.48-7.99)$ & 3.10 & $(0.01-5.03)$ & 3.65 & $(1.12-10.88)$ \\
\hline Cardiogenic shock (yes vs no) & 12.58 & $(3.25-20.77)$ & 1.40 & $(0.16-12.61)$ & 6.53 & $(2.00-19.64)$ \\
\hline Congestive heart failure (yes vs no) & 3.77 & $(1.10-13.45)$ & 2.91 & $(0.82-10.24)$ & 3.76 & $(1.37-8.92)$ \\
\hline $\operatorname{HbA1c}(\%)$ & 1.28 & $(1.01-2.68)$ & 2.87 & $(1.56-4.72)$ & 1.81 & $(1.03-3.00)$ \\
\hline Type of bypass (arterial vs venous) & 0.39 & $(0.22-0.53)$ & 0.67 & $(0.47-0.81)$ & 0.57 & $(0.30-0.74)$ \\
\hline Ischaemic time ( $\mathrm{min})$ & 1.00 & $(0.97-1.03)$ & 1.01 & $(0.94-1.08)$ & 1.00 & $(0.97-1.03)$ \\
\hline Cardiopulmonary bypass time (min) & 1.01 & $(0.98-1.02)$ & 0.99 & $(0.96-1.04)$ & 1.00 & $(0.98-1.02)$ \\
\hline Pre-operation LVEF (\%) & 0.96 & $(0.93-1.00)$ & 0.94 & $(0.89-1.00)$ & 0.96 & $(0.93-0.98)$ \\
\hline Post-operation LVEF (\%) & 0.98 & $(0.93-1.04)$ & 0.98 & $(0.93-1.03)$ & 1.00 & $(0.97-1.05)$ \\
\hline ICU stay (days) & 1.01 & $(0.98-1.05)$ & 1.05 & $(1.02-1.09)$ & 1.04 & $(1.02-1.06)$ \\
\hline
\end{tabular}

CAD: coronary artery disease, CABG: coronary artery bypass grafting, LVEF: left ventricular ejection fraction

independent Student's $t$-testing was applied for discrete or continuous variables, respectively. The Kaplan-Meier method was used to estimate the cumulative survival of diabetic patients after CABG surgery. Multiple Cox regression was used to investigate the independence of factors associated with all-cause mortality after CABG surgery when variables were significant by univariate analysis. Subjects were considered as censored cases if the outcomes were not available. A $p$-value of $<0.05$ was considered statistically significant. The results are presented as means \pm standard deviations (SDs).

\section{RESULTS}

\subsection{Baseline Characteristics}

Table 1 shows the comparison of baseline characteristics between males and females among diabetic patients with CAD after CABG surgery. The females differed from the males in the following ways. Females had a lower proportion of smoking $(11.5 \%$ vs $27.5 \%)$, alcohol consumption (6.8\% vs. $14.1 \%)$, arterial bypass received $(68.9 \%$ vs $79.9 \%)$ and a lower CAD number $(2.74 \pm 0.54$ vs $2.83 \pm 0.44)$ than males. Females had a higher proportion of hypertension $(78.7 \%$ vs $71.8 \%)$ and renal failure $(20.9 \%$ vs $14.1 \%)$, were older $(66.50 \pm 8.18 \mathrm{yr}$ vs $64.02 \pm 10.05 \mathrm{yr})$, had a longer duration of diabetes $(14.78 \pm 5.32 \mathrm{yr}$ vs $12.64 \pm 4.85 \mathrm{yr})$, had higher FPG $(201.2 \pm 106.6 \mathrm{mg} / \mathrm{dl}$ vs $169.1 \pm 107.6 \mathrm{mg} / \mathrm{dl})$, SBP
(141.1 $\pm 22.2 \mathrm{mmHg}$ vs $135.0 \pm 20.7 \mathrm{mmHg}$ ) and cholesterol $(204.3 \pm 131.8 \mathrm{mg} / \mathrm{dl}$ vs $189.3 \pm 44.3 \mathrm{mg} / \mathrm{dl})$, had longer days of ICU stays $(8.64 \pm 13.88$ yr vs $6.44 \pm 7.17)$, and had better preoperative LVEF $(54.09 \pm 19.91 \%$ vs $50.93 \pm 19.68 \%$ ).

\subsection{Survival Rates, Deaths, and Causes of Death}

One hundred and five out of 816 study patients died during the 7-year follow-up. Of these deaths. The major causes of death were septic shock (35.2\%) and heart failure (29.5\%). As Figure 1 shows, the 1-month, 1-year, 3-year, 5-year, and 7-year cumulative survival among diabetic patients with CAD after CABG surgery were 95.96\% (95\% confidence interval (CI): 94.61-97.31\%), 92.83\% (95\%CI: 91.04-94.62\%), 88.80\% (95\%CI: $86.51-$ $91.09 \%), 85.56 \%$ (95\%CI: $83.33-87.79 \%)$, and $83.51 \%$ (95\%CI: 80.12-86.90\%), respectively. A statis- tical significance $(p=0.04)$ of gender difference was found for seven-year cumulative survival after CABG surgery (males: $86.01 \%$ vs females: $77.65 \%$ ).

\subsection{Risk Factors for Mortality}

The effect of independently associated risk factors upon all-cause mortality among diabetic patients with CAD after CABG was examined using the multiple Cox regression models. As is depicted in Table 2, subsequent to 


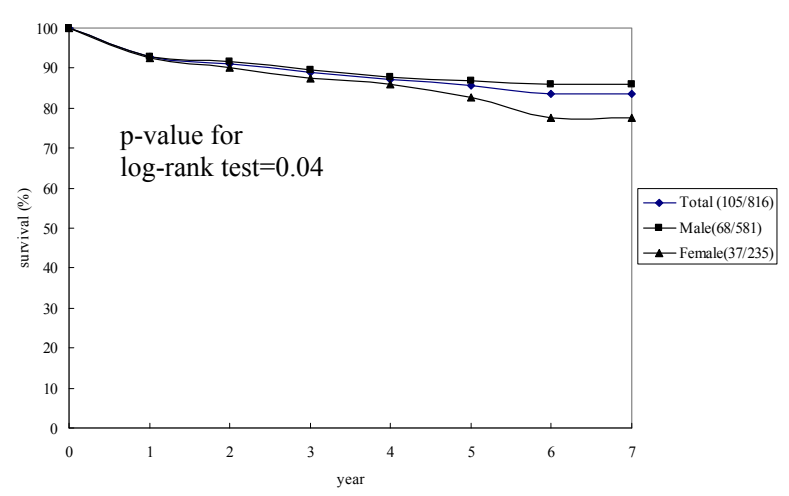

Figure 1. Seven-year cumulative survival among type 2 diabetic patients with CAD after CABG surgery in males versus females.

adjustment for confounding factors, gender (male vs. female, $\mathrm{RR}=0.26,95 \% \mathrm{CI}$ : $0.13-0.54)$, age at surgery $(\mathrm{RR}=1.05,95 \% \mathrm{CI}: 1.00-1.10)$, respiratory failure (yes vs. no, $\mathrm{RR}=3.65$, 95\%CI: 1.12-10.88), cardiogenic shock (yes vs. no, $\mathrm{RR}=6.53,95 \% \mathrm{CI}$ : 2.00-19.64), congestive heart failure (yes vs. no, $\mathrm{RR}=3.76,95 \% \mathrm{CI}$ : 1.37-8.92), HbAlc $(\mathrm{RR}=1.81$, 95\%CI: 1.03-3.00), type of bypass (arterial vs venous, $\mathrm{RR}=0.57,95 \% \mathrm{CI}$ : $0.30-0.74$ ), preoperative LVEF (RR=0.96, 95\%CI: 0.93-0.98), and days of ICU stay ( $R R=1.04,95 \% \mathrm{CI}$ : 1.02-1.06) appeared to be statistically significantly related to all-cause mortality. The dramatically different results of multiple Cox regression analyses of the data stratified by gender also show in Table 2.

\section{DISCUSSION}

\subsection{Implications of Gender Difference as Regards Associated Risk Factors for All-Cause Mortality}

Diabetes is known as a determinant of prognosis in CAD and may pose an increased risk for major complications during CABG surgery [3,18]. Diabetic patients represent about $20 \%$ of patients undergoing CABG surgery [19]. Although previous studies have shown that diabetic patients with $\mathrm{CAD}$ have worse long-term survival after CABG than non-diabetics $[1,2]$, the plausible biological mechanisms that could link diabetes to the early and late outcome of CABG remain uncertain. The extent of vascular compromise in diabetes is of particular relevance in CAD patients, as it might account for the more severe coronary artery pathology and might also impair the biological integrity of the bypass grafts [3,20,21]. Preserved functional and structural properties of CABG conduits are essential for a successful myocardial revascularization [3,21].

The continuing surgical and technical improvements in the operative management of CAD patients who undergo
CABG surgery, as well as consistently better anesthetic and postoperative care, might have made it difficult to identify any specific gender-related risk factors associated with all-cause mortality. However, our results showed that diabetic females with CAD who were referred for $\mathrm{CABG}$ surgery clearly differed from males in that they were older and had a higher proportion of hypertension and renal failure. This finding not only implies that females were at a later disease stage or had more serious symptoms than males before being referred for CABG surgery, but also that associated chronic diseases were major risk indicators for females with CAD [17,22]. In addition, females more frequently had renal dysfunction. To some extent, this might be explained by older age and more frequent histories of personal disease such as diabetes mellitus and hypertension [22].

In the present study, female diabetic patients with CAD had slightly elevated all-cause mortality during the 7-year study when compared to male patients. Our findings are in agreement with previous experiences focused on all CAD population that showed gender differences in late mortality $[17,22]$. They are also in agreement with a large study taking major risk factors into account that showed that there were no substantial differences in the long-term survival of males and females after CABG surgery [22]. Although there seem to be only small differences overall in long-term survival after CABG surgery between males and females, there may be greater gender differences in specific patient subgroups. But after adjustment for confounding factors, female sex was still independently associated with all-cause mortality in the current study. This difference between male and female may become of greater importance than in earlier times because the population of patients undergoing CABG surgery has aged, there are more patients with abnormal pre- and postoperative LVEF and the patient populations have more diffuse diseases [16,22].

It should be noted that a difference in survival rates in females and males occurs after CABG surgery about 4 to 5 years in this study. Previous studies have also shown that after adjustment for all independent predictors of long-term mortality, female sex was an independent predictor of improved 5-year survival [23]. While the information yielded by univariate analyses was valuable, the relationships between predictors and all-cause mortality among diabetic patients with CAD after CABG surgery were far too complex to be answered completely by univariate analysis. It has been well documented in many academic studies that females presenting for CABG have a vastly different preoperative risk profile than that of their male counterparts [23]. The risk profiles of males and female are quite different and indicate that although it is usually appropriate to directly compare the two groups, male and female, with respect to a specific predictor, the predictor might be inappropriate. The use of multivariate models is particularly important because they fully ac- 
count for all related important risk factors. In this study, some risk factors for all-cause mortality were detrimental to males and others were more detrimental to females after CABG surgery. Greater adverse preoperative characteristics such as diabetic females being older and having more associated diseases can be suggested as one explanation of the higher mortality rates among female. Since female gender was an independent predictor of all-cause mortality in the multivariate analysis, discrepancies in preoperative demographic or clinical characteristics could only explain part of the increased mortality rates among diabetic females after CABG surgery. However, dissimilarities in profiles between female and male diabetic patients might contribute to the debate about the gender difference in outcome after CABG surgery [23,24]. In addition, increased mortality among female patients was probably due to the fact that females had more comorbid conditions than did men at the time of referral, perhaps because they were not being evaluated aggressively enough [25]. From the viewpoint of preventive medicine, although discrepancies suggest that associated predictive variables for long-term survival after CABG surgery differ between hospitals and could change with time, regular identification of risk factors makes it possible to focus attention on current problems to minimize surgical risk and reduce mortality, especially among females. Clinical decision making and follow-up should also not be influenced by stereotypes but by specific findings.

\subsection{Methodological Considerations}

Although based on the follow-up study design, that is, being prospective in design as well as including all diabetic patients with CAD who underwent CABG surgery in a well defined patient population for 7 years, we not only determined the hospital mortality, but also further explored the cumulative survival and prognostic factors related to all-cause mortality among diabetic patients with CAD after CABG surgery. The present study still has some limitations that need to be considered while assessing the results. First, some of the clinical variables obtained from medical records could have resulted in misclassification bias. Nevertheless, it seems reasonable to assume that if misclassification bias was present, it was not related to gender and all-cause mortality, thus could be viewed as nondifferential misclassification. Second, potential Berkson's bias (selection bias) was inevitable due to the hospital-based study design. This population was not only not exactly representative of the whole general population, but it was also difficult to estimate the survival of a matched total population sample in Taiwan, preferably in the region of Taipei. Third, due to having no information on the quantity and duration of some risk factors such as smoking and alcohol drinking, we could not clarify the dose-response effect between specific personal habits and all-cause mortality. Fourth, the female sample size might not be large enough to identify the exact confidence interval of related prognostic factors. Further studies should be conducted with larger sample sizes for female to re-examine gender-specific prognostic factors for long-term survival among diabetic patients with CAD after CABG surgery.

\section{CONCLUSIONS}

The all-cause mortality of diabetic patients with CAD after CABG surgery and several gender-related differences pertaining to all-cause mortality were investigated, including the relationships between all-cause mortality and smoking, myocardial infarction, cardiogenic shock, respiratory failure, congestive heart failure, older age at surgery, emergent operation, pulmonary edema, and days of ICU stay. In order to increase long-term survival among diabetic patients with $\mathrm{CAD}$, adequate diagnosis and treatment risk factors after CABG surgery are essential.

\section{ACKNOWLEDGEMENTS}

This study was presented partly in the $2^{\text {nd }}$ International Congress on "PREDIABETES and the METABOLIC SYNDROME", Barcelona, Spain, 2007.

\section{REFERENCES}

[1] T. Hakala, O. Pitkanen, P. Halonen, et al., (2005) Early and late outcome after coronary bypass surgery in diabetic patients. Scand Cardiovasc J, 39(3), 177-181.

[2] A. M. Calafiore, M. Di Mauro, G. Di Giammarco, et al., (2003) Effect of diabetes on early and late survival after isolated first coronary bypass surgery in multivessel disease. J Thorac Cardiovasc Surg, 125(1), 144-154.

[3] V. H. Thourani, W. S. Weintraub, B. Stein, et al., (1999) Influence of diabetes mellitus on early and late outcome after coronary artery bypass grafting. Ann Thorac Surg, 67(4), 1045-1052.

[4] T. Ono, J. Kobayashi, Y. Sasako, et al., (2002) The impact of diabetic retinopathy on long-term outcome following coronary artery bypass graft surgery, J Am Coll Cardiol, 40(3), 428-436.

[5] J. D. Lin, W. B. Shieh, M. J. Huang, et al., (1993) Diabetes mellitus and hypertension based on the family history and 2-h postprandial blood sugar in the Ann-Lo district (Northern Taiwan). Diabetes Res Clin Pract, 20(1), 75-85.

[6] C. L. Li, S. T. Tsai, P. Chou, et al., (1999) Comparison of the results between two diagnostic criteria by ADA and WHO among subjects with FPG 5.6-7.8 mmol/l in Kin- $\mathrm{Hu}$ and Kin-Chen, Kinmen, 1991-94. Diabetes Res Clin Pract, 45(1), 51-59.

[7] P. Chou, M. J. Liao, H. S. Kuo. et al., (1994) A population survey on the prevalence of diabetes in Kin-Hu, Kinmen. Diabetes Care, 17(9), 1055-1058.

[8] P. Chou, C. L. Li, H. S. Kuo, et al., (1997) Comparison of the prevalence of two diabetes surveys between 1987-88 
and 1991-92 in Pu-Li, Taiwan. Diabetes Res Clin Pract, 38(1), 61-67.

[9] H. D. Chen, C. K. Shaw, W. P. Tseng, et al., (1997) Prevalence of diabetes mellitus and impaired glucose tolerance in Aborigines and Chinese in eastern Taiwan. Diabetes Res Clin Pract, 38(3), 199-205.

[10] F. H. Lu, Y. C. Yang, J. S. Wu, et al., (1998) A population-based study of the prevalence and associated factors of diabetes mellitus in southern Taiwan. Diabetes Med, 15(7), 564-572.

[11] K. T. Chen, C. J. Chen, E. W. Gregg, et al., (1999) High prevalence of impaired fasting glucose and type 2 diabetes mellitus in Penghu Islets, Taiwan: evidence of a rapidly emerging epidemic? Diabetes Res Clin Pract, 44(1), 59-69.

[12] World Health Organization, (1999) Definition, diagnosis and classification of diabetes mellitus and its complications: Report of a WHO Consultation, Part 1, Diagnosis and classification of diabetes mellitus, Geneva, World Health Organization.

[13] Joint National Committee on Prevention, Detection, Evaluation, and Treatment of High Blood Pressure, (1997) The sixth report of the joint national committee on prevention, detection, evaluation, and treatment of high blood pressure. Arch Intern Med, 157(21), 2413-2446.

[14] C. M. Liu, T. H. Tung, J. H. Liu, et al., (2005) A community-based epidemiological study of elevated serum alanine aminotransferase levels in Kinmen, Taiwan. World J Gastroenterol, 11(11), 1616-1622.

[15] C. H. Huang, S. T. Lai, and Z. C. Weng, (2001) Risk factors for mortality in primary isolated coronary artery bypass grafting surgery. J Formos Med Assoc, 100(5), 299-303.

[16] C. H. Huang, C. P. Hsu, S. T. Lai, et al., (2004) Operative results of coronary artery bypass grafting in women. Int $\mathrm{J}$ Cardial, 94(1), 61-66.

[17] M. C. Hsiung, J. Wei, C. Y. Chang, et al., (2006) Long- term survival and prognostic implications after coronary artery bypass grafting in Chinese patients with coronary artery disease. Acta Cardiol, 61(5), 519-524.

[18] W. Whang and J. T. Bigger, (2000) Diabetes and outcomes of coronary artery bypass graft surgery in patients with severe left ventricular dysfunction: results from the $\mathrm{CABG}$ Patch trial database, The CABG Pateh Trial Investigators and Coordinators. J Am Coll Cardiol, 36(4), 1166-1172.

[19] C. Rajakaruna, C. A. Rogers, C. Suranimala, et al., (2006) The effect of diabetes mellitus on patients undergoing coronary surgery: A risk-adjusted analysis. J Thorac Cardiovasc Surg, 132(4), 802-810.

[20] R. Lorusso, S. Pentiricci, R. Raddino, et al., (2003) Influence of type 2 diabetes on functional and structural properties of coronary artery bypass conduits. Diabetes, 52(11), 2814-2820.

[21] J. G. Motwani and E. J. Topol, (1998) Aortocoronary saphenous cein graft disease: Pathogenesis, predisposition and prevention. Circulation, 97(9), 916-931.

[22] G. Brandrup-Wognsen, H. Berggren, M. Hartford, et al., (1996) Female sex is associated with increased mortality and morbidity early, but not late, after coronary artery bypass grafting. Eur Heart J, 17(9), 1426-1431.

[23] I. K. Toumpoulis, C. E. Anagnostopoulos, S. K. Balaram, et al., (2006) Assessment of independent predictors for long-term mortality between women and men after coronary artery bypass grafting: Are women different from men? J Thorac Cardiovasc Surg, 131(2), 343-351.

[24] C. D. Naylor and C. M. Levinton, (1993) Sex-related difference in coronary revascularization practices: the perspective from a Canadian queen management project. Can Med Assoc J, 149(7), 965-973.

[25] M. R. Williams, A. F. Choudhri, D. L. Morales, et al., (2000) Gender differences in patients undergoing coronary artery bypass surgery, from a mandatory statewide database. J Gend-Specif Med, 3(1), 41-48. 